\title{
COMMISSION 26: DOUBLE STARS (ÉTOILES DOUBLES)
}

Président: J. Dommanget. Secrétarre: O. G. Franz.

\author{
Comptes Rendus des Séances
}

\section{Première Séance}

Avant de passer à l'ordre du jour, l'assemblée, sous la présidence de J. Dommanget, désigne le secrétaire de séance. Le choix se porte sur $O$. G. Franz, qui accepte d'en assumer la charge.

La Commission respecte ensuite quelques instants de silence pour honorer la mémoire de deux de ses membres: C. E. Luplau-Janssen et G. B. van Albada, ainsi que d'un ancien membre: J. Dick, tous trois récemment décédés.

Le Président signale que la Commission a dû enregistrer avec grand regret, l'arrêt de l'inlassable activité de trois de ses meilleurs observateurs: W. H. van den Bos (72000 mesures), P. Baize (40000 mesures) et W S. Finsen (15000 mesures dont 6000 mesures interférométriques de couples particulièrement difficiles. Il leur exprime une fois de plus, au nom de la Commission, sa plus profonde gratitude pour le travail accompli.

Etant donné la faible participation que l'on pouvait craindre depuis longtemps à la présente Assemblée Générale et l'impossibilité de prendre aucune décision valable dans une telle situation, le Président a décidé d'interroger tous les membres de la Commission par une lettre circulaire du 13 juin 1973, sur les principaux points prévus alors à l'ordre du jour de la Commission. Sur ses 38 membres, 15 membres absents aujourd'hui ont répondu, ce qui, avec les 7 membres présents à la séance, permet d'assurer une majorité de 22 votes valables. Il fut précisé par ailleurs que l'absence de réponse serait considérée comme une approbation implicite des décisions qui seraient prises sur la base des votes effectifs. A la demande du Président, l'assemblée approuve le processus.

Le Président signale aussi avoir reçu de la plupart des quinze membres absents ayant répondu à la lettre-circulaire, des souhaits de fructueux travaux pour la Commission. Il les en remercie vivement.

\section{Changement de NOM De la COMmission No. 42 (Photometric Binaries)}

Ce point de l'ordre du jour est examiné en tout premier lieu afin de permettre au Président de la Commission No. 42, M. Plavec, de présenter son point de vue et de participer à l'éventuelle discussion qui s'en suivra, avant d'assister à d'autres réunions.

Le Président signale que la nouvelle dénomination proposée pour cette commission (Close Binaries) a suscité la réticence unanime des 15 membres cités plus haut, cette dénomination ayant une signification différente pour les observateurs d'étoiles doubles visuelles. M. Plavec démontre la nécessité absolue pour sa Commission d'abandonner l'ancienne dénomination, laquelle ne recouvre plus son activité actuelle, puisqu'elle porte sur les couples réellement serrés, qu'ils soient observables photométriquement par suite d'une forte inclinaison orbitale ou qu'ils soient seulement observables spectroscopiquement. Après un échange de vues entre les membres présents, la Commission décide de ne pas formuler d'opposition à cette proposition de changement de nom, mais de faire connaître au Secrétaire Général, ses objections et ses regrets devant l'expression choisie.

M. Plavec remercie la Commission pour sa compréhension.

\section{COMPOSITION DE LA COMMISSION}

Sur proposition du Président et en application de l'article 20b, chapitre III des 'Directives' de l'Union, sont considérés comme démissionnaires: B. Cester, O. J. Eggen et G. P. Kuiper, ces collègues n'ayant pas répondu à plusieurs lettres les invitant à faire connaître leur désir de poursuivre 
leur activité au sein de la Commission. Toutefois, dans le cas de B. Cester, sur proposition de M. G. Fracastoro, le statu quo sera provisoirement observé.

Le Président fait part de la proposition du Comité Organisateur sortant de considérer comme nouveaux membres d'une part: A. A. Kiselev et P. S. The, membres de l'Union et d'autre part: R. S. Harrington, P. J. Morel, R. E. Nather, G. A. Starikova et R. L. Walker, pour autant que ces chercheurs soient élus membres de l'Union sur recommandation de leurs comités nationaux respectifs.

Ces propositions sont adoptées.

La Commission choisit ensuite son nouveau Comité d'Organisation dont la composition sera soumise au Comité Exécutif de l'Union pour approbation. La proposition est issue de concertations entre les membres du Comité sortant, visant à une représentation aussi variée que possible des diverses spécialités existant au sein de la Commission, tout en respectant un minimum de continuité dans sa gestion.

W. Heintz ayant, pour des raisons personnelles à caractère scientifique, décliné l'offre de faire partie du Comité d'Organisation, sont proposés: A. H. Batten, P. C. Couteau, A. N. Deutsch, J. Dommanget, O. G. Franz, S. L. Lippincott, P. Muller et K. Aa. Strand.

Pour la Présidence et la Vice-Présidence, le Comité d'Organisation sortant propose respectivement: Miss S. L. Lippincott et $P$ Muller.

Ces propositions sont chacune adoptées malgré deux votes défavorables que leurs auteurs ont justifiés auprès du Président par une opposition au mode d'élection du Bureau et du Comité d'Organisation, insuffisamment démocratique à leur avis.

L'un d'eux, F. Holden a adressé à ce sujet une lettre-circulaire (14 juillet 1973) à tous les membres de la Commission, les priant de faire connaître au Président avant l'Assemblée Générale, leur éventuelle approbation d'une réforme consistant dans des élections au scrutin secret ('written ballot'). Seules 5 réponses ont été reçues: elles comportent une abstention, deux réponses favorables et deux autres entièrement opposées à la proposition. Une des réponses favorables (U. GüntzelLingner) comporte par ailleurs une proposition d'amendement.

Le Président estime qu'un large débat sur cette importante question est souhaitable et que, pour lui donner une solution précisément aussi démocratique qu'on la réclame, la présence d'un grand nombre de membres est exigé. Aussi, propose-t-il de renvoyer cette question aux séances de la Commission lors de la prochaine Assemblée Générale et de la traiter, comme le conçoit P. Muller, dans le cadre d'un règlement d'ordre intérieur. Indépendamment d'ailleurs, le Secrétaire Général vient de prier les Commissions d'établir un tel règlement conformément à l'article 22 du 'Règlement' de l'Union.

Comme bien d'autres présidents de commissions, J. Dommanget estime toutefois qu'il faudra éviter l'établissement de règles trop strictes, mais bien plutôt rechercher la mise au point de principes de fonctionnement démocratiques. De telles règles risqueraient de compliquer sinon de bloquer le fonctionnement de la Commission.

Le Président transmettra à tous les membres de la Commission, une copie des documents reçus à ce sujet du Secrétaire Général. Comme le souhaitent K. Aa. Strand et A. H. Batten, la mise au point d'un projet à discuter à la prochaine Assemblée Générale, sera laissée aux soins du nouveau Comité d'Organisation et du nouveau Bureau.

L'ensemble de ce processus est approuvé par les membres présents.

\section{REPORT ON ASTRONOMY}

Le rapport établi par le Président sur la base des informations reçues des membres de la Commission et publié dans les Transactions XVA, est adopté sans modification, compte tenu des votes des membres absents.

\section{Priorités en astronomie}

Le texte publié sur les priorités dans le même volume, à la suite du Rapport signalé au point précédent, est également approuvé sans modification et dans les mêmes conditions de vote. 


\section{Prochain colloque}

Par la lecture d'une lettre du Prof. A. Poveda, Directeur de l'Observatorio Astronómico de l'Universidad Nacional Autonoma de Mexico, le Président fait part de l'invitation de celui-ci à tenir le prochain colloque sur les étoiles doubles et multiples, à Mexico City en octobre 1974.

La Commission approuve l'idée d'un tel colloque mais estime cependant préférable d'en reporter la date à l'automne 1975. Elle est reconnaissante au Prof. A. Poveda pour son invitation et l'en remercie vivement.

\section{Publication des mesures}

Le Président rend compte de sa négociation avec les éditeurs de Astronomy and Astrophysics destinée à résoudre les problèmes que la publication des mesures avait fait surgir à une certaine époque. Il a été convenu que celles-ci seraient généralement publiées-ainsi que la plupart des orbites-dans les Supplement Series, pour des raisons d'ordre pratique et budgétaire et qu'il serait tenu compte des recommandations de la commission en cette matière. L'accord conclu avec les éditeurs de la revue a fait l'objet d'une publication dans la Circulaire d'Information No. 58-annexe (nov. 1972) de la Commission, éditée par P. Muller. Il prévoit également un groupement, dans la mesure du possible, des séries de mesures et des calculs d'orbites dans un minimum de fascicules.

De tels accords pourraient être passés avec d'autres grandes revues astronomiques où sont publiées des mesures d'étoiles doubles et des orbites, afin d'assurer la régularité de leur publication mais aussi le respect des recommandations de la Commission (rappelées dans la circulaire citée).

La Commission approuve cette suggestion. J. Dommanget est chargé de poursuivre sa tâche auprès de Astronomy and Astrophysics, cette revue ayant demandé que la Commission désigne officiellement son représentant. Le nouveau Président, S. L. Lippincott pourrait jouer un rôle identique auprès des grandes revues astronomiques américaines.

\section{Recommandations (voir Transactions XVA, p. 309)}

Voir ci-dessus: 6.

\section{(a) Publication des Mesures}

\section{(b) Révision de l'Index Catalogue}

La Commission marque un vif intérêt au projet de C. E. Worley d'une révision de l'Index Catalogue, consistant dans l'adjonction des couples nouveaux et dans la recherche systématique des erreurs qu'il contient. P. C. Couteau estime particulièrement satisfaisant, le délai de cinq ans proposé pour l'achèvement du travail, le survey qu'il poursuit en collaboration avec P. Muller devant alors toucher à sa fin.

Comme proposé dans une récente lettre par C. E. Worley, la Commission décide de ne pas entamer dès maintenant une discussion approfondie des modalités d'exécution de ce travail, mais de procéder par lettre-circulaire, pour connaître les suggestions de chacun.

Le Président félicite C. E. Worley pour le travail déjà accompli (la recherche des erreurs et leur correction a porté sur les $\frac{3}{4} \mathrm{du}$ travail et a nécessité le traitement de quelque 300000 cartes). Il remercie le U.S. Naval Observatory et son directeur, K. Aa. Strand de mettre à la disposition de C. E. Worley, le personnel et les moyens nécessaires à sa tâche.

\section{(c) Diffusion des Publications}

La Commission adopte le voeu de J. Dommanget de voir chacun de ses membres adresser, dans la mesure du possible, des tirés-à-part de ses travaux à tous ses collègues de la Commission. 


\section{(d) Terminologie Relative à la Parallaxe Dynamique}

Parmi les réponses reçues des membres absents au sujet de cette recommandation, certaines expériment des remarques ou objections qu'il y aurait lieu de discuter devant une assemblée plus vaste. Aussi, sur proposition de l'auteur même de la recommandation, la Commission décide d'en reporter l'examen à la prochaine Assemblée Générale

J. Dommanget profite toutefois de l'occasion pour rappeler que sa proposition porte principalement sur la distinction essentielle à faire entre les parallaxes dynamiques orbitales et non-orbitales, sous peine de voir le crédit accordé aux parallaxes dynamiques, très sérieusement affecté. Trop d'astronomes ne connaissent pas cette distinction et portent dès lors un jugement regrettable sur la qualité des parallaxes orbitales car ils les confondent avec celles données par exemple par $H$. N. Russell et C. E. Moore dans le tableau 53 de 'The Masses of the Stars'. Ces dernières sont en effect nonorbitales, c'est-à-dire calculées sur une base statistique tenant compte seulement du mouvement relatif des composantes en un point de la trajectoire, en l'absence d'orbite connue.

\section{Seconde Séance}

\section{SituAtion DANS L'HÉmis Phère sud (voir: Transactions XVA, p. 310)}

Le Président rend compte des contacts suivis qu'il a eus tant avec le Council for Scientific and Industrial Research d'Afrique du Sud qu'avec les autorités de la University of Michigan (U.S.A.) concernant l'arrêt des programmes d'observation d'étoiles doubles respectivement au Republic Observatory à Johannesbourg et au Lamont-Hussey Observatory à Bloemfontein.

Comme aucun représentant officiel de chacune de ces deux institutions n'est présent à Sydney, sa proposition de constituer une délégation chargée d'un échange de vues avec eux, perd tout son sens et est donc retirée, malgré l'appui qu'elle avait trouvé auprès des membres absents ayant fait connaître leur position à ce sujet.

Par ailleurs, la proposition de F. Holden de constituer une sous-commission chargée d'étudier le problème cie l'observation dans l'hémisphère sud et de prendre toute disposition nécessaire pour mettre en route de nouveaux programmes, n'a pas rencontré l'approbation des membres de la Commission. Seules cinq réponses ont été reçues à la lettre de F. Holden du 14 juillet 1973, parmi lesquelles deux seulement sont favorables. La Commission estime donc que son activité dans ce domaine ne réclame pas la mise en place d'une telle sous-commission.

Le Président souligne les appels du Comité d'Organistion de la Commission auprès de tous les organismes astrònomiques de l'hémisphère sud pour qu'ils prennent conscience de l'importance de l'observation des couples visuels.

Au sujet de l'Afrique du Sud, il ressort d'informations reçues tant du Directeur de l'Observatoire Sud-Africain, Sir R. Woolley, que du Président du CSIR, le Dr F. J. Hewitt et confirmées au cours de la présente Assemblée Générale par le Prof. G. G. Cillie, chef de la délégation sud-africaine, que la station de Johannesbourg sera maintenue en fonctionnement pour l'instant. G. Knipe serait prêt à y reprendre les observations de binaires visuelles dès la fin de l'année en cours.

Par ailleurs, M. KJerk envisagerait l'observation de couples visuels au Bosscha Observatory à Lembang (Indonésie) ce qui ramenerait un grand réfracteur au service des binaires visuelles, comme le souhaite son Directeur, B. Hidajat.

P. R. Hurly a fait savoir qu'il dispose à l'Université đu Cap, d'un "area scanner' construit par Warner et Nather et qu'il compte entreprendre un programme photométrique d'observation des couples visuels en UBV.

A l'Observatoire de Cordoba, G. M. Iannini s'occupe de la mise au point d'un équipement devant lui permettre l'observation photographique de couples visuels à l'aide du télescope de $154 \mathrm{~cm}$ de Bosque Alegre.

R. R. de Freitas Mourão précise son intérêt pour l'échange d'astronomes entre observatoires de manière à profiter au maximum des meilleures conditions climatiques dans chacun d'eux. 
Aucune information précise ne peut malheureusement être donnée sur la destination du réfracteur du Lamont-Hussey Observatory situé près de Bloemfontein.

\section{QUESTIONS SCIENTIFIQUES}

La séance se termine par les trois exposés suivants:

A. H. Batten: Orbit and Period of $\beta 1163$ (en cours d'impression dans: Journal of the Royal Astronomical Society of Canada);

A. M. Sinzi: Measurement of Double Stars by Occultations with Small Telescopes (en cours de publication dans: Report of Hydrographic Researches, Tokyo, No. 8, 1974);

W J. Luyten: Double Stars with Common Proper Motion (voir: Proper Motion Survey with the 48 in. Schmidt Telescope, No. 25 et 29, University of Minnesota, Minneapolis).

\section{Erratum}

à

'Reports on Astronomy'

p. 298, deuxième ligne à partir du bas de la page:

lire: 'réflecteurs' au lieu de: 'réfracteurs' 